\title{
Análisis de eficiencia del sector industrial manufacturero en cinco países suramericanos, 1995-2008
}

\author{
Efficiency analysis of the manufacturing industry in five \\ south american countries, 1995-2008
}

Recibido: 25 de septiembre de 2014 - Revisado: 28 de abril de 2015 - Aceptado: 28 de agosto de 2015

Adriana María Molina Romero

Geovanny Castro Aristizábal ${ }^{3}$

\section{Resumen}

Este artículo calcula la eficiencia técnica de cinco países suramericanos en la industria manufacturera durante 1995-2008. A través de un análisis de frontera estocástica se estima la eficiencia para probar si la aglomeración contribuye a acercar más a su frontera de productividad eficiente. Se emplea una función de producción translogarítmica, porque permite cambios tecnológicos y variaciones en las proporciones de insumos y productos de la elasticidad de escala. Los resultados muestran que en el sector manufacturero no se generaron ni mejoramientos en la eficiencia técnica ni cambios tecnológicos durante los catorce años. La evidencia sugiere que las variables ambientales determinadas por la formación bruta de capital fijo total o solo en maquinaria y equipo influyen en el comportamiento del sector.

\section{Palabras clave}

Eficiencia técnica, frontera estocástica, industria manufacturera.

\begin{abstract}
This article measures the technical efficiency of five south american countries in manufacturing during 1995-2008. Through an analysis of stochastic frontier efficiency is estimated to test whether agglomeration contributes to get efficient productivity closer to its border. Translog production function is used, because it allows technological changes and variations in the proportions of inputs and outputs of the elasticity of scale. The results show that in the manufacturing sector improvements in technical efficiency and technological changes were not generated during these fourteen years. Evidence suggests that environmental variables determined by the gross formation of total fixed capital or only in machinery and equipment influence the behavior of the sector.
\end{abstract}

\section{Keywords}

Technical efficiency, stochastic frontier, manufactuting industry.
${ }^{1}$ El presente artículo es resultado de trabajo de grado para optar al título de magíster en Economía de la Pontificia Universidad Javeriana. Cali, Colombia.

${ }^{2}$ Economista de la Pontificia Universidad Javeriana, Cali, Colombia; y consultora económica en ONU-Hábitat.

Correo electrónico:

adrimolir@hotmail.com

${ }^{3}$ Economista (M.S.) y Ph.D. (c) de la Universidad de Zaragoza. Zaragoza, España. Profesor del Departamento de Economía y miembro del Grupo de Investigación en Desarrollo Regional (GIDR) de la Pontificia Universidad Javeriana. Cali, Colombia.

Correo electrónico: gcastro@javerianacali.edu.co

Para citar este artículo use: Molina, A., \& Castro, G. (2015). Análisis de eficiencia del sector industrial manufacturero en cinco países suramericanos, 1995-2008. Revista Civilizar Ciencias Sociales y Humanas, 15(29), 93-112. 


\section{Introducción}

Por años, las industrias manufactureras en los países en vía de desarrollo han conducido a la generación de empleo y al crecimiento de la actividad económica, gracias a su dinamismo tanto hacia el interior como hacia otros sectores (Moreno, 2008). Su desempeño está determinado por la productividad, que es la relación entre los insumos y el producto, dada una tecnología, siendo evaluada por la eficiencia. Usualmente, la eficiencia se representa a través de una frontera de producción que constituye la máxima producción alcanzable en la industria (Mutis, 2006). Por consiguiente, la vinculación de las economías aglomeradas en ese "estado ideal" permite países más eficientes productivamente y más desarrollados, porque ahora comparten sus infraestructuras, mano de obra especializada, spillovers de conocimiento, entre otros aspectos (Acevedo, 2004; Grether, 1999).

El análisis de fronteras de producción estocásticas, es uno de los tipos de medición del desempeño, que presenta, a modo de ventaja, la realización de estimaciones de funciones de producción que incluyen las industrias técnicamente ineficientes (Brescia, Lema, \& Barrón, 2003). Para el caso de la industria manufacturera, dicha frontera tiene en cuenta como variables determinantes: a) la producción, que se mide con el valor bruto de producción, b) la mano de obra, calculada por el total de personas ocupadas en el sector, c) el capital, medido por el consumo intermedio de cada país y d) el nivel de tecnología, que se estima por la inversión en capital fijo o por la formación bruta de capital fijo (FBKF), esperando encontrar una relación positiva entre la eficiencia del sector y la aglomeración de los cinco países suramericanos en vía de desarrollo (Mutis, 2006).

Por otro lado, la medición del cambio de la productividad total de los factores (TFP, por su sigla en inglés) y su importancia en uno o varios sectores, son temas de interés para muchos autores, porque consideran no solo la contribu- ción de los factores productivos sino también los elementos que afectan tanto positiva como negativamente al funcionamiento de la economía. El problema radica en que existen muy pocas aplicaciones empíricas orientadas hacia un país o un grupo de países en vía de desarrollo, en particular en sectores que demandan mano de obra como la manufacturera.

En aras de la medición, se busca realizar comparaciones con economías similares. En el caso de las naciones de la Comunidad Andina (CAN), objeto del presente trabajo, estas poseen características semejantes. Todas consiguieron independizarse de la corona española, tienen el mismo idioma y algunas lenguas precolombinas. Geográficamente, son vecinas fronterizas y están comunicadas por la cadena montañosa de los Andes, la segunda más alta del mundo. En su conjunto, el número de habitantes es de 124.905 .914 y la superficie terrestre de $4.718 .322 \mathrm{~km}^{2}$, representando el $16 \%$ del total de Suramérica ${ }^{1}$. Cuentan con un sistema de gobierno democrático y su cercanía territorial les permite menores costos en las exportaciones. Así mismo, sus preferencias, costumbres y cultura facilitan las negociaciones, lo que les ayuda a mantenerse como socios comerciales. Sin embargo, no han conseguido la misma dinámica económica que Brasil y Chile (Casas, 2001).

De hecho, con base en información de la CAN para el año 2008, las participaciones del sector industrial manufacturero en el producto interno bruto (PIB) son similares (Venezuela 15 $\%$, Perú $14,5 \%$, Colombia 13,9 \%, Ecuador 9,2 $\%$ y Bolivia 9,1\%). En promedio, el PIB manufacturero de estos países ha variado significativamente en el último quinquenio. Para el año 2005 , dicho sector creció en 5,6\%, aumentando 1,4 puntos porcentuales en el año 2006, llegando a un crecimiento del 7,9 \% en el 2007 y a una caída del 4,5 \% para el año 2008. Así mismo, el impacto que tiene este sector sobre el nivel del empleo es relevante. De acuerdo con la CAN, entre los años 2001 y 2008, el coeficiente de co- 
rrelación, que explica la relación entre el número de personas ocupadas y el PIB de la industria manufacturera, fue de 98,5\% para Colombia, seguido por Ecuador (98,2 \%), Perú (88,3 \%), Bolivia $(85,3 \%)$ y Venezuela $(65,8 \%)^{2}$.

Ahora bien, la correcta medición de los cambios en la TFP hace posible conocer si las economías aglomeradas contribuyen a acercarse a la frontera eficiente. Aunque no hay que olvidar que si bien en su conjunto, estos países suramericanos pueden tener técnicas de producción similares, difieren demográfica, geográfica y políticamente, factores que pueden intervenir en la eficiencia del sector.

Por lo anterior, este artículo responde a cuáles son los componentes y fuentes de variación de la productividad de factores en la industria manufacturera para Bolivia, Colombia, Ecuador, Perú y Venezuela durante el periodo 1995-2008, realizando estimaciones de la eficiencia técnica (ET) a través de un análisis de frontera estocástica (SFA, por su sigla en inglés). Conserva la siguiente estructura: después de esta parte introductoria, se presenta el marco de referencia e investigaciones orientadas a la eficiencia y su medición en el contexto nacional e internacional. Luego se expone la metodología empleada y finalmente se exhiben los resultados, el análisis de los modelos estimados, las conclusiones y las referencias bibliográficas.

\section{Marco de referencia e investigaciones empíricas nacionales e internacionales}

La competitividad de un país se relaciona con la productividad de su industria. En la medida que la industria se fortalece productivamente, se favorece el paso de actividades simples hacia actividades más complejas que generan mayores rentas y que están más ligadas al desarrollo tecnológico y a la innovación. Estos beneficios potenciales son incluso superiores por el rápido cambio tecnológico, la mayor apertura de mercados y la fragmentación e internacionalización de la producción, convir- tiendo al comercio de los productos en el medio más idóneo para "transportar" tecnología, para afianzar competitividad relativa y para lidiar con la globalización (Vera, 2009) ${ }^{3}$.

Ahora, el concepto de eficiencia se utiliza en las empresas para adelantar los procesos productivos que determinan en buena parte su competitividad y sus posibilidades de supervivencia (Quintero, 2005). A su vez, el comportamiento y funcionamiento de las firmas impulsan a tomar decisiones óptimas basándose principalmente en el supuesto de la racionalidad de los agentes económicos.

Los expertos en aspectos económicos consideran que una situación de máxima eficiencia, puede ocurrir bajo el concepto de eficiencia paretiana, donde no se puede mejorar la situación de un agente sin empeorar la de otro. Esta concepción, propuesta por Pareto (1906), ha avanzado hasta llegar al punto de facilitar otras clases de eficiencias como son: la eficiencia económica, ET, eficiencia de asignación, eficiencia de costos y eficiencia de escala.

Por ende, al referirse a la eficiencia se piensa directamente en la TFP, definida como el cociente entre el índice de productos y el índice de insumos. Sin embargo, cuando se emplean conjuntos de insumos y productos, la TFP se puede asociar a la eficiencia asignativa (EA) y la ET (Patiño, Gómez, \& Osorio, 2010). La EA relaciona la "mejor" asignación óptima de insumos o productos de la empresa dado los precios de los mismos y la tecnología. Mientras que la ET, refleja la capacidad que una firma tiene para producir el máximo producto con un conjunto dado de insumos. Este concepto lo introdujo en la economía Koopmans (1951), quien entiende que un punto en el espacio de bienes (finales o intermedios) es eficiente, si al alcanzar un mayor nivel de output en alguno de los bienes, se recurre a la disminución del nivel de output en cualquiera de los otros. De igual forma, Debreu (1951) esboza un método para 
medirla a través de un índice que denominó coeficiente de la utilización de recursos (Castro \& Salazar, 2011), aunque el principal referente sobre el tema de la medición es Farrell (1957).

Extendiendo la medición de la eficiencia, sobre todo en la técnica ${ }^{4}$, Patiño et al. (2010) elaboraron una clara visión de la medición de la eficiencia orientada hacia los insumos con base en Coelli, Rao y Battese (2005), donde Farrell (1957) ilustró su idea en un ejemplo sencillo con empresas que emplean dos insumos $x_{1}$ y $x_{2}$ para producir un solo producto $q$, desde el supuesto de rendimientos constantes a escala. En concreto, si se conoce la isocuanta $S S^{\prime}$ ' como las diferentes combinaciones de factores que proporcionan eficientemente el único bien $q$ (véase gráfica 1), se permite medir la ET como la relación de la combinación eficiente con respecto a la combinación ineficiente de insumos para producir un nivel de producto $q$, tal como lo muestra la ecuación (1).

$$
E T=0 Q / 0 p
$$

La ecuación (1) corresponde al cociente entre las distancias $\mathrm{O} Q$ y $\mathrm{O} P$ y representa las diferentes combinaciones de factores que proporcionan eficientemente el único nivel de producción del bien $q$ (véase gráfica 1), por lo que se permite medir la ET como la relación entre la combinación eficiente $(\mathrm{O} Q)$ con respecto a la combinación ineficiente de insumos $(\mathrm{O} P)$ para producir un nivel de producto $q$.

Entonces, si una determinada compañía usa cantidades de insumos representadas por el punto $P$ para producir una unidad de $q$ (dado a lo largo de la isocuanta $S S^{\prime}$ ), la ineficiencia técnica de la firma puede representarse por la distancia de $Q P$. Esta distancia muestra que una empresa produce el mismo nivel de producto $q$ que en el punto $Q$. No obstante, está empleando una combinación de insumos $\left(x_{1 P}\right.$ y $\left.x_{2 P}\right)$ mayor, por lo tanto, a esta firma se le considera ineficiente, o lo que es lo mismo, puede producir el mismo nivel en el punto $Q$, con una combinación de insumos $\left(x_{1 Q}\right.$ y $\left.x_{2 Q}\right)$ menor a la empleada en el punto $P$ (véase gráfica 1 ).

La ineficiencia es por lo común expresada en términos porcentuales y será igual a la relación ${ }^{Q P} / 0 P$. Esta proporción representa la cantidad de insumos que se deben reducir para alcanzar la eficiencia; en particular, si $\frac{Q P}{O P} \rightarrow 0$, la firma es más eficiente.

\section{Eficiencia técnica orientada al insumo}

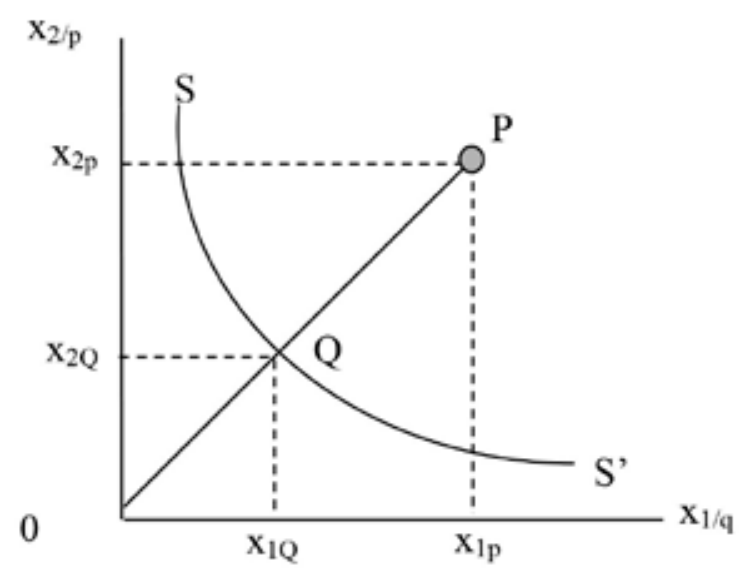

Fuente: Patiño et al., 2010, p. 92.

En contraste, la medida de ET puede expresarse en términos de la ineficiencia a través de la distancia $Q P$. Como los porcentajes de eficiencia y de ineficiencia deben sumar uno, y considerando que esta última está medida por ${ }^{Q P} /{ }_{\mathrm{O} P}$, entonces $E T=1-{ }^{Q P} /{ }_{\mathrm{OP}}$. Ahora bien, como $Q P=O P-O Q$ y suponiendo que ${ }^{Q P /} \mathrm{OP}=1$, entonces la eficiencia se puede considerar de la forma que muestra la ecuación (2).

$$
E T=1-\frac{Q P-0 Q}{0 P}=1-1+\frac{0 Q}{0 P}=\frac{0 Q}{0 P}
$$

Esta ET toma valores entre cero y uno; por lo tanto, proporciona el grado de ET de la firma. Lo que significa que, si $E T=1$, la firma es completamente eficiente y entre más cercana a cero más se aleja de la eficiencia. La medida de ET orientada a dos insumos y un solo producto, 
puede emplearse también en múltiples insumos con múltiples productos, que se manifiesta en términos de función de distancias expresadas en (3) donde $d_{i}(x, q)$ representa la función de distancia de insumos y $x$ el vector de insumos con los cuales se obtiene el vector de producción $q$. La empresa es técnicamente eficiente ${ }^{5}$ si se encuentra en la frontera, en cuyo caso $E T=1 \mathrm{y}$ $d_{i}(x, q)=1$.

$$
E T=1 / d_{i}(\mathbf{x}, \mathbf{q})
$$

Después de exponer las medidas de eficiencias propuestas por Farrell (1957), donde asume como dada la función de producción, es indispensable encontrar el método para estimarlas. El autor, a partir de la noción empírica de la función de producción ${ }^{6}$, generó desde un diagrama de dispersión una frontera eficiente con datos observables (véase gráfica 2-b). Luego, la estimación de esta frontera puede realizarse a partir de: múltiples insumos y múltiples productos, "orientada a la producción” (véase gráfica 2-a); un producto con una combinación de insumos, "orientada al insumo" (véase gráfica 2-b), o un solo insumo con un conjunto de productos, "orientada al producto" (véase gráfica 2-c).

Gráfica 2

\section{La frontera eficiente, casos para su estimación}

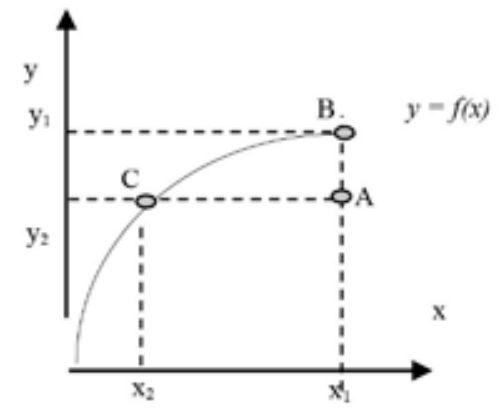

a. Según función de producción

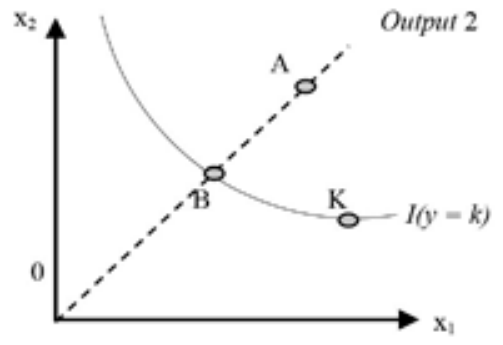

b. Según isocuanta

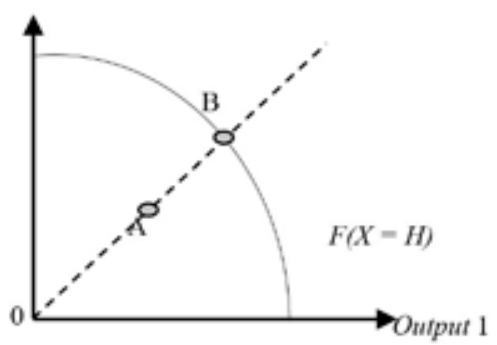

c. Según FPP

Fuente: Castro \& Salazar, 2011, p. 96.

Este trabajo se centrará en la estimación de la frontera de producción orientada al insumo, debido a que relaciona la producción de un único sector (un producto) con un conjunto de insumos (capital, mano de obra, cambio tecnológico, FBKF y maquinaria y equipo). Así, siguiendo a Castro y Salazar (2011), la medición guiada hacia los insumos (véase gráfica 2-b), muestra que la producción eficiente se sitúa en el punto $B$ de la frontera $I(y=k)$, porque utiliza la proporción necesaria de recursos para un nivel específico de producción $k$; esto equivale a que la ET sea el ratio $\rho=O B / O A$. Situación contraria para la unidad $A^{7}$.

Ahora bien, las estimaciones se realizarán a partir del SFA, tal y como lo sugieren Coelli et al. (2005). Esta técnica asume una forma funcional dada para la relación entre los insumos y el producto. Fue expuesta inicialmente por Meeusen y Vanden (1977) y aplicada por Aigner, Lovell y Schmidt (1977), Coelli, Rao y Battese (1998) y Kumbhakar (2000) $)^{8}$. El modelo del SFA se define teniendo en cuenta la función de producción como punto de partida (Sharma, Sylwester, \& Margono, 2007).

$$
\operatorname{Ln} Y_{i t}=\operatorname{Lnf}\left(x_{i t}, t, \beta\right)-U_{i t}
$$

Donde $Y_{i t}$ representa el producto de los $i$-th países en el tiempo $t$. A esta frontera se le adiciona el término de error aleatorio al componente determinístico: 


$$
\operatorname{Ln} Y_{i t}=\operatorname{Lnf}\left(x_{i t}, t, \beta\right)-\varepsilon_{i t} \operatorname{con} \varepsilon_{i t}=V_{i t}-U_{i t}, U_{i t} \geq 0
$$

Donde $i=1,2, \ldots, m$ denota los países y $t$ indica el horizonte de tiempo y sirve como una aproximación para el cambio tecnológico. Ahora, el vector $x_{i t}$ comprende los insumos del i-ésimo país en el tiempo $t, \beta$ el vector de coeficientes de estimación y $\varepsilon_{i t}$ el término de error estocástico, el cual está compuesto por dos variables independientes una de la otra; $V_{i t}$ denota un error bilateral del ruido estadístico con distribución $N \sim\left(0, \sigma_{v}^{2}\right)$ y $U_{i t}$ señala por una parte el término de error representando la ineficiencia técnica9.

El presente documento, como se mencionó, estimará la ecuación del modelo (5) con orientación al insumo, dada la especificación translogarítmica - ecuación (6)-, que será expuesta en la parte metodológica,

$$
\begin{gathered}
\operatorname{Lnf}\left(x_{i t}, t, \beta\right)=\beta_{0}+\beta_{1} \operatorname{LnK}_{i t}+\beta_{2} \operatorname{Ln} L_{i t}+\beta_{3} t+0,5\left[\beta_{4}\left(\operatorname{Ln} K_{i t}\right)^{2}+\beta_{5}\left(\operatorname{LnL} L_{i t}\right)^{2}+\beta_{6} t^{2}\right] \\
+\beta_{7} \operatorname{Ln} K_{i t} \operatorname{Ln} L_{i t}+\beta_{8} t \operatorname{Ln} K_{i t}+\beta_{9} t \operatorname{Ln} L_{i t}
\end{gathered}
$$

Donde los insumos son capital, $K$, la mano de obra, $L$, el cambio tecnológico está sujeto al tiempo y la función de producción depende de la variación de este.

Finalmente, por varios años, algunos autores han visto la necesidad de conocer la eficiencia de un país con respecto a uno o más sectores económicos. La literatura que se asocia con la medición, reporta diversas metodologías de eficiencia que son aplicables para los diferentes escenarios que posee cada estudio, siendo el que más se maneja la medición técnica. En la tabla 1 se muestran las investigaciones empíricas sobre la medición de eficiencia que han sido implementadas al sector de la industria manufacturera de Europa, Asia y América Latina (Colombia, México y Uruguay). Así mismo, ilustra la metodología empleada y los principales resultados $\mathrm{y} / \mathrm{o}$ conclusiones a las que llegan los autores.

\begin{tabular}{|c|c|c|c|}
\hline Autor(es) & País(es) & Metodología & Resultados o conclusiones \\
\hline Castro y Salazar (2011) & Colombia & $\begin{array}{l}\text { Eficiencia financiera utilizando el análisis } \\
\text { envolvente de datos a través de los modelos } \\
\text { de rendimientos a escala constante y va- } \\
\text { riable (Constant Returns of scale - CCR-y } \\
\text { Variable Returns of scale - BCC - respec- } \\
\text { tivamente) }\end{array}$ & $\begin{array}{l}\text { La ineficiencia de las empresas del sec- } \\
\text { tor se debe a un exceso de gastos de ad- } \\
\text { ministración y a su baja razón corriente. } \\
\text { Las firmas líderes en ventas son las más } \\
\text { eficientes }\end{array}$ \\
\hline Patiño et al. (2010) & Colombia & Análisis de frontera estocástica & $\begin{array}{l}\text { Únicamente cuatro de las } 24 \text { empresas } \\
\text { alcanzaron una eficiencia técnica superior } \\
\text { al } 90 \%\end{array}$ \\
\hline Díaz y Sánchez (2008) & España & $\begin{array}{l}\text { Función de producción de frontera estocás- } \\
\text { tica y determinantes de la ineficiencia me- } \\
\text { diante panel no balanceado }\end{array}$ & $\begin{array}{l}\text { Las empresas pequeñas y medianas tien- } \\
\text { den a ser menos ineficientes que las gran- } \\
\text { des compañías, por el bajo grado de com- } \\
\text { plejidad en la organización empresarial y } \\
\text { control de gestión }\end{array}$ \\
\hline Margono y Sharma (2006) & Indonesia & $\begin{array}{l}\text { Eficiencia técnica a través de un modelo de } \\
\text { frontera }\end{array}$ & $\begin{array}{l}\text { El tipo de propiedad contribuye a la inefi- } \\
\text { ciencia en el sector textil }\end{array}$ \\
\hline
\end{tabular}

\section{Tabla 1}

\section{Estudios empíricos del sector manufacturero}




\begin{tabular}{|c|c|c|c|}
\hline Autor(es) & País(es) & Metodología & Resultados o conclusiones \\
\hline Acevedo (2004) & Colombia & $\begin{array}{l}\text { Eficiencia técnica en el ámbito nacional por } \\
\text { medio del análisis de fronteras estocásticas }\end{array}$ & $\begin{array}{l}\text { Existe una relación positiva entre la con- } \\
\text { centración industrial y la eficiencia en la } \\
\text { producción, al menos a un nivel agregado } \\
\text { por departamento }\end{array}$ \\
\hline Batra y Tan (2003) & $\begin{array}{l}\text { México } \\
\text { Colombia } \\
\text { Indonesia } \\
\text { Malasia } \\
\text { Guatemala } \\
\text { Taiwán }\end{array}$ & $\begin{array}{l}\text { Niveles de eficiencia a partir de la función } \\
\text { frontera de producción estocástica, y com- } \\
\text { parar las distribuciones de eficiencia entre } \\
\text { firmas de diferentes tamaños }\end{array}$ & $\begin{array}{l}\text { La eficiencia técnica aumenta con el ta- } \\
\text { maño de la empresa y así mismo, se iden- } \\
\text { tifica un conjunto de factores que distin- } \\
\text { gue a las firmas más eficientes }\end{array}$ \\
\hline Kim (2003) & Corea & $\begin{array}{l}\text { Funciones de producción de frontera esto- } \\
\text { cásticas mediante un panel no balanceado } \\
\text { de empresas }\end{array}$ & $\begin{array}{l}\text { El tamaño de la empresa, las exportacio- } \\
\text { nes y las actividades de investigación y } \\
\text { desarrollo, tienen efecto positivo y sig- } \\
\text { nificativo en todos los sectores; mientras } \\
\text { que el capital extranjero parece no afectar } \\
\text { los niveles de eficiencia }\end{array}$ \\
\hline $\begin{array}{l}\text { Green, Harris y Mayes } \\
\qquad(1991) \\
\text { Caves (1990) } \\
\text { Green y Mayes (1989) } \\
\text { Harris (1989) }\end{array}$ & $\begin{array}{l}\text { Australia y } \\
\text { Reino Unido } \\
\text { Estados } \\
\text { Unidos }\end{array}$ & $\begin{array}{l}\text { Técnicas paramétricas y fronteras } \\
\text { estocásticas para cuantificar la ineficiencia } \\
\text { técnica }\end{array}$ & $\begin{array}{l}\text { A pesar de las diferencias en los niveles } \\
\text { de productividad las fronteras de inefi- } \\
\text { ciencia son similares }\end{array}$ \\
\hline Mini y Rodríguez (2000) & Filipinas & Eficiencia técnica & $\begin{array}{l}\text { Los aumentos de la eficiencia técnica de- } \\
\text { bilitan las políticas de las pyme, además, } \\
\text { las exportaciones y las intervenciones del } \\
\text { gobierno se asocian positivamente con la } \\
\text { eficiencia, aunque la relación entre el apo- } \\
\text { yo del gobierno y la eficiencia técnica es } \\
\text { algo más débil }\end{array}$ \\
\hline Grether (1999) & México & Eficiencia para un conjunto de empresas & $\begin{array}{l}\text { El capital extranjero tiene influencia po- } \\
\text { sitiva en la eficiencia en el ámbito de la } \\
\text { planta, sin embargo, no genera efectos } \\
\text { spillovers en el escenario sectorial }\end{array}$ \\
\hline Tansini y Triunfo (1998) & Uruguay & $\begin{array}{l}\text { Eficiencia técnica, por la función de pro- } \\
\text { ducción de frontera estocástica }\end{array}$ & $\begin{array}{l}\text { El nivel promedio de eficiencia se incre- } \\
\text { mentó en dos ramas industriales: pin- } \\
\text { turas, barnices, y lacas y en artículos de } \\
\text { limpieza y tocador, se mantuvo estable en } \\
\text { matanza y preparación de carnes y aves y } \\
\text { disminuyó en productos farmacéuticos y } \\
\text { medicamentos }\end{array}$ \\
\hline
\end{tabular}

Fuente: elaboración propia.

\section{Metodología}

La información de las variables estructurales en la industria manufacturera de la CAN permitirá estimar la función de producción véase ecuación (6)-, considerando el nivel de producción como variable dependiente y como variables explicativas el capital, mano de obra (insumos) y cambio tecnológico. La intención es aportar un modelo para evaluar la ET del sector y la productividad total de factores de los cinco países suramericanos en su conjunto, sin olvidar que la existencia de otros factores, que no se incluyeron en este estudio, tales como las variables financieras y comerciales, puede alterar dicha estimación. Además, los resultados obtenidos aquí contribuirán a ampliar la relativa escasez de la literatura para los países en vía de desarrollo. 
Para la estimación del modelo, con orientación al insumo, se usará el método de máxima verosimilitud $\mathrm{y}$, siguiendo a Coelli et al. (2005), se emplearán fronteras estocásticas desde las metodologías de Aigner et al. (1977) y Meeusen y Vanden (1977), mediante un panel de datos no balanceados, permitiendo modelar no solo el término de error aleatorio normal del modelo, sino también una variable aleatoria no observable asociada con la ineficiencia técnica de la producción $U_{i t}$, aplicándolo en el programa computable FRONTIER 4.1 del Centro de Eficiencia y Análisis de Productividad.

Con base en la función (6), se calculan los efectos marginales de los insumos y el tiempo (cambio tecnológico) sobre el nivel de producción que corresponden a:

$$
\begin{aligned}
& \frac{1}{f} \Delta f=\beta_{1} \frac{1}{K} \Delta K+0,5 * 2 \beta_{4}(\operatorname{LnK}) \frac{1}{K} \Delta K+\beta_{7} \operatorname{LnL} \frac{1}{K} \Delta K+\beta_{8} t \frac{1}{K} \Delta K \\
& \frac{1}{f} \Delta f=\left(\beta_{1}+\beta_{4}(\operatorname{LnK})+\beta_{7} L n L+\beta_{8} t\right) \frac{1}{K} \Delta K \\
& \frac{\Delta f}{f} * 100=\left[\left(\beta_{1}+\beta_{4}(\operatorname{Ln} K)+\beta_{7} \operatorname{Ln} L+\beta_{8} t\right) \frac{\Delta K}{K}\right] * 100 \\
& \Delta \% f=\left(\beta_{1}+\beta_{4}(\operatorname{LnK})+\beta_{7} \operatorname{Ln} L_{+} \beta_{8} t\right) \Delta \% K \\
& \frac{\Delta \% f}{\Delta \% K}=\left(\beta_{1}+\beta_{4}(\operatorname{LnK})+\beta_{7} \operatorname{Ln} L+\beta_{8} t\right)
\end{aligned}
$$

Por analogía, para cambios en el nivel de trabajo y cambios en $t$ :

$$
\begin{aligned}
& \frac{\Delta \% f}{\Delta \% L}=\left(\beta_{2}+\beta_{5} L n L+\beta_{t} \operatorname{Ln} K+\beta_{9} t\right) \\
& \frac{\Delta \% f}{\Delta t}=100 *\left(\beta_{3}+\beta_{6} t+\beta_{8} L n K+\beta_{9} \operatorname{LnL}\right)
\end{aligned}
$$

Así, las expresiones (7) y (8) son las elasticidades insumo-producto del capital y del trabajo, en su orden, y el resultado en (9) indica el cambio porcentual que se da en el nivel de producto por un cambio en la unidad de tiempo. Cuando las unidades de capital y de trabajo son iguales a uno, y el periodo de tiempo es cero, entonces estas elasticidades están dadas por $\beta_{l}$ y $\beta_{2}$ respectivamente, y el efecto del cambio tecnológico sobre la variación porcentual del producto es $100 \beta_{3}$, coeficientes que se estiman e interpretan en la sección cuarta.

Por consiguiente, se considerará como punto de partida la función de producción translogarítmica (6) porque es una de las formas funcionales más usadas, ya que permite retornos no-constantes a escala, cambios tecnológicos y variaciones en las proporciones de insumos y productos de la elasticidad de escala. Dado que el enfoque central es la ET y la TFP, se siguen dos argumentos:

1) El propuesto por Battese y Coelli (1988), en el cual la ET de la unidad ith se define como $T E_{i t}=E\left[\exp \left(-U_{i}\right) \mid \varepsilon_{i t}\right]$, donde $\varepsilon_{i t}=$ $V_{i t}-U_{i t}$, para las ith países en el tiempo $t$.

Sustituyendo (6) en (5) se obtiene la frontera de producción translogarítmica que se estima por máxima verosimilitud ${ }^{10}$. Por lo tanto, dicha estimación define la $T E_{i t}$ como: 


$$
T E_{i t}=E\left(e^{-u_{i t}} \mid \varepsilon_{i t}\right)=\frac{\varnothing\left[\left(\mu_{*},_{i t}\right) /\left(\sigma_{*}\right)-\sigma\right]_{*}}{\varnothing\left(\mu_{*_{i t}} / \sigma *\right)} \exp \left(-\mu_{*_{i t}}+\frac{1}{2} \sigma_{*}\right)
$$

Donde:

$$
\mu_{*}=\frac{-\sigma^{2} u \varepsilon+\delta z \sigma_{v}^{2}}{-\sigma_{u}^{2}+\sigma_{v}^{2}} \quad \mathrm{y} \quad \sigma_{*}^{2}=\frac{\sigma_{u}^{2} \sigma_{v}^{2}}{\sigma_{u}^{2}+\sigma_{v}^{2}}
$$

2) El que plantean Sharma et al. (2007), quienes obtienen la producción total de factores con solo cantidades siguiendo a Kumbhakar y Lovell (2000).

El diferencial total de $\operatorname{Ln} f\left(x_{i \ell}, t, \beta\right)$ en (4) con respecto al tiempo de producción es:

$$
\frac{d \operatorname{Ln} f(x, t, \beta)}{d t}=\frac{\partial \operatorname{Lnf}(x, t, \beta)}{\partial t}+\sum_{j} \frac{\partial \operatorname{Lnf}(x, t, \beta)}{\partial x_{j}} \frac{d x_{j}}{d t}
$$

El primer término en el lado derecho de (12) mide los cambios en la producción causados por la frontera de progreso tecnológico (TP). Para la función de producción translogarítmica de (6) estos corresponden a:

$$
T P_{i t}=\beta_{3}+\beta_{6} t+\beta_{8} \operatorname{Ln}\left(K_{i t}\right)+\beta_{9} \operatorname{Ln}\left(L_{i t}\right)
$$

El resultado en (13) corresponde al obtenido en (9). Ahora, el segundo término en el lado derecho de (12) puede ser escrito como:

$$
\sum_{j} \frac{\partial \operatorname{Lnf}(x, t, \beta)}{x_{j}} \frac{d x_{j}}{d t}=\sum_{j} e_{j} x_{j}
$$

Con $e_{j}$ como la elasticidad de producción del $j t h$ insumo $\left(e_{j}=\partial \operatorname{Lnf} / \partial x_{j}\right)$ y $x_{j}$ es el cambio del $j$ th insumo a través del tiempo. Las elasticidades de producción con respecto al capital y a la mano de obra de la función de producción de la translogarítmica (6) están dadas por las expresiones (7) y (8). Dado lo anterior, el diferencial total de la función de producción con respecto al tiempo de producción queda como muestra la ecuación (15).

$$
\dot{y}=\frac{d \operatorname{Ln} f(x, t, \beta)}{d t}-\frac{d u}{d t}=T P=\sum_{j} e_{j} \dot{x}_{j}+\Delta T E
$$

Donde $\Delta T E=\partial u / \partial t$ es el cambio de la ET. Así, de (15) el cambio de la producción global no solo se ve afectado por el TP, y los cambios en el uso de insumos, sino también en la ET. Para examinar el efecto de $T P$ y $\triangle T E$ en $\triangle T F P$, se define $T F P$ como el crecimiento de la producción no explicado por el crecimiento de los insumos:

$$
\dot{T F P}=\dot{y}-\sum_{j} s_{j} \dot{x}_{j}
$$


Así, $s_{j}$ aporta el j’s insumo en el costo de producción. Mediante la sustitución de (16) en (15), se obtiene la ecuación (17).

$$
\dot{T F P}=T P+\Delta T E+(e-1) \sum_{j} \lambda_{j} \dot{x}_{j}+\sum_{j}\left(\lambda_{j}-s_{j}\right) \dot{x}_{j}
$$

Donde $e=\sum e_{j}$ denota la medición de los rendimientos a escala y $\lambda_{j}=e / e$ es la relación entre la elasticidad de producción de la jth insumos con respecto a los rendimientos a escala. Aumentando los insumos de producción en igual proporción, la producción crecerá en mayor magnitud si hay un incremento en los retornos de escala y en menos con la disminución de los rendimientos a escala. Si hay retornos a escala constantes, $e=1$ y los cambios en la cantidad de insumos hacen que no afecte los cambios en el factor total de productividad. Cuando la información del precio no está disponible para determinar los costos, el componente de eficiencia en la asignación, el último término en (17), no puede ser calculado empíricamente. Siguiendo a Kumbhakar y Lovell (2000), se asume que $\mathrm{s}_{\mathrm{j}}=e_{\mathrm{j}} / e$ para toda $j$ $\mathrm{y}$, así (17) se reduce a:

$$
T \dot{F P}=T P+\Delta T E+(e-1) \sum_{j} \frac{e_{j}}{e} \dot{x}_{j}
$$

\section{Resultados}

\section{Estadísticas descriptivas generales.}

En América Latina, el valor agregado industrial durante 1995 y 2005 obtuvo una alta participación en valor total, tal fue el caso de Brasil con el $36 \%$, seguido de México con $28 \%$ y Argentina que creció $13 \%$. Así mismo, Venezuela con un $6 \%$, Chile y Colombia ambos con tasas de
$4 \%$, Perú, Ecuador y Bolivia con participaciones menores al $5 \%$ (Amézquita, 2008). Sin embargo, un panorama del PIB manufacturero de Colombia, Perú, Bolivia, Venezuela y Ecuador en los últimos años viene experimentando un crecimiento lento según cifras de la CAN (véase gráfica 3).

\section{Gráfica 3}

Producto interno bruto del sector manufacturero, 1995-2008 (millones de dólares)

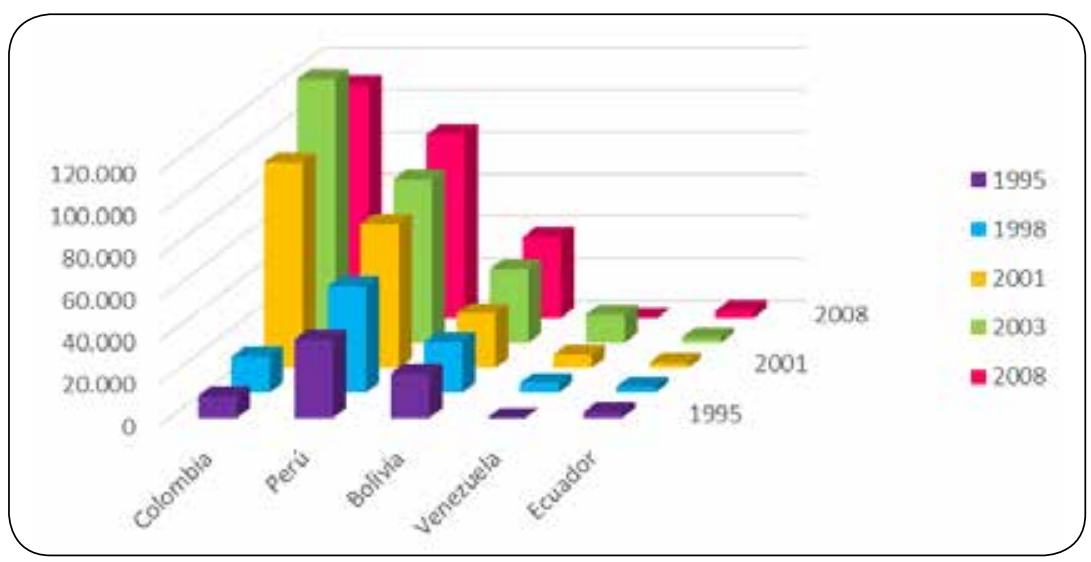

Fuente: elaboración propia con base en el Compendio de series estadísticas de la CAN, 2011 
Al descomponer el PIB nacional por rama de actividad, como deja ver la gráfica 4 , durante el año 2008, se presenta de forma conjunta una concentración principalmente en las actividades de: otros servicios, manufactura, impuestos a los productos netos y comercio al por mayor $\mathrm{y}$ al por menor. Seguidas por otras actividades no menos importantes para la economía como son: minería e hidrocarburos, administración pública y defensa, intermediación financiera, hoteles y restaurantes, construcción, transportes y almacenamiento, agricultura, ganadería, caza y silvicultura, suministro de electricidad, gas y agua y petróleo (solo en Venezuela). Aunque no quiere decir, que cada país sea el más participativo según el orden mostrado anteriormente.

Gráfica 4

Participación \% del producto interno bruto nacional por rama de actividad, 2008

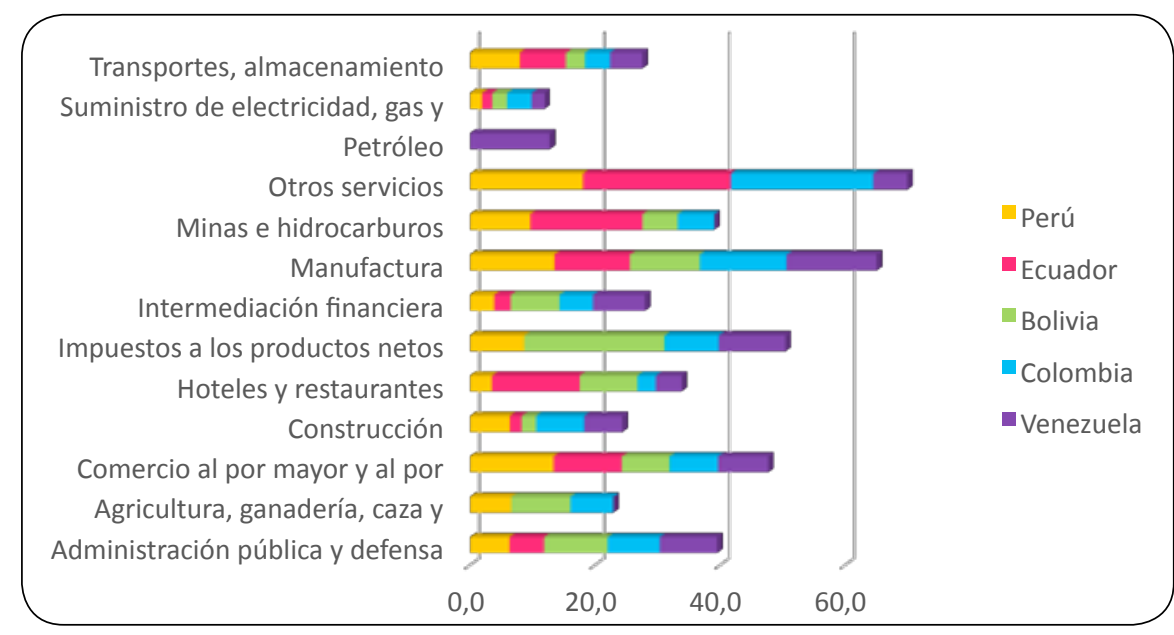

Fuente: elaboración propia con base en el Compendio de series estadísticas de la CAN, 2011

Este crecimiento paulatino en el producto del sector industrial coincide con el bajo progreso del personal ocupado que reporta la CAN para los últimos años. La gráfica 5 permite observar con más amplitud la evolución de las personas ocupadas en el sector manufacturero por país, donde Colombia ha reflejado un crecimiento sostenido desde el año 2001, caso similar al ocurrido en Ecuador desde el 2003, a pesar de que los otros países no conservan la misma tendencia porque su PIB manufacturero no ha variado tan significativamente como los dos casos en mención.

Gráfica 5

Personas ocupadas en el sector manufacturero, 1995-2008 (miles de personas)
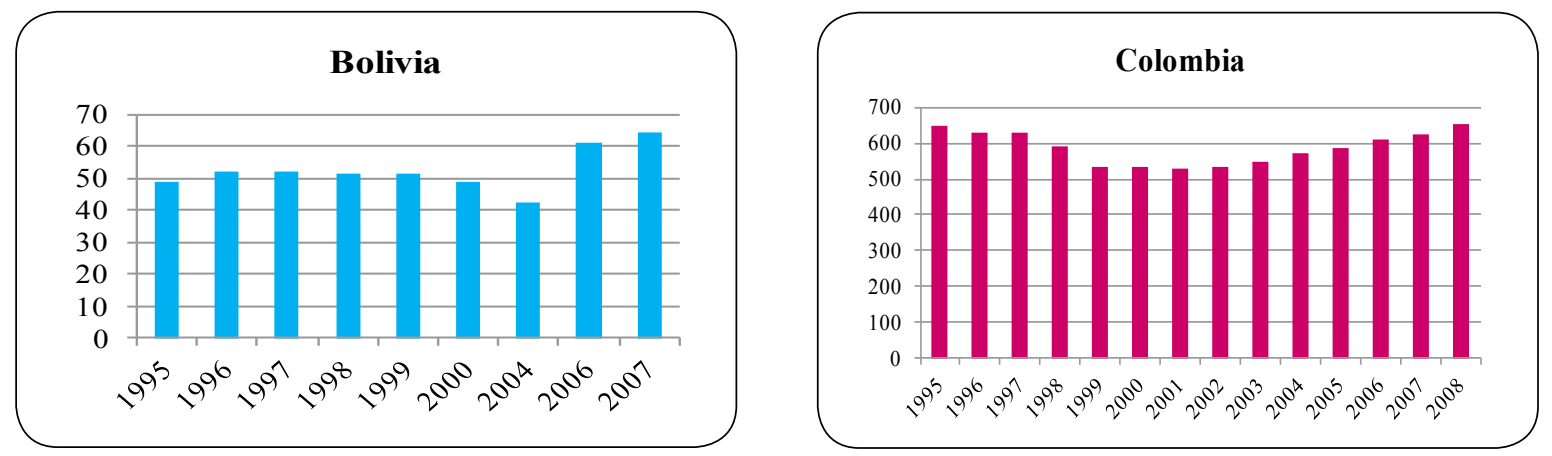

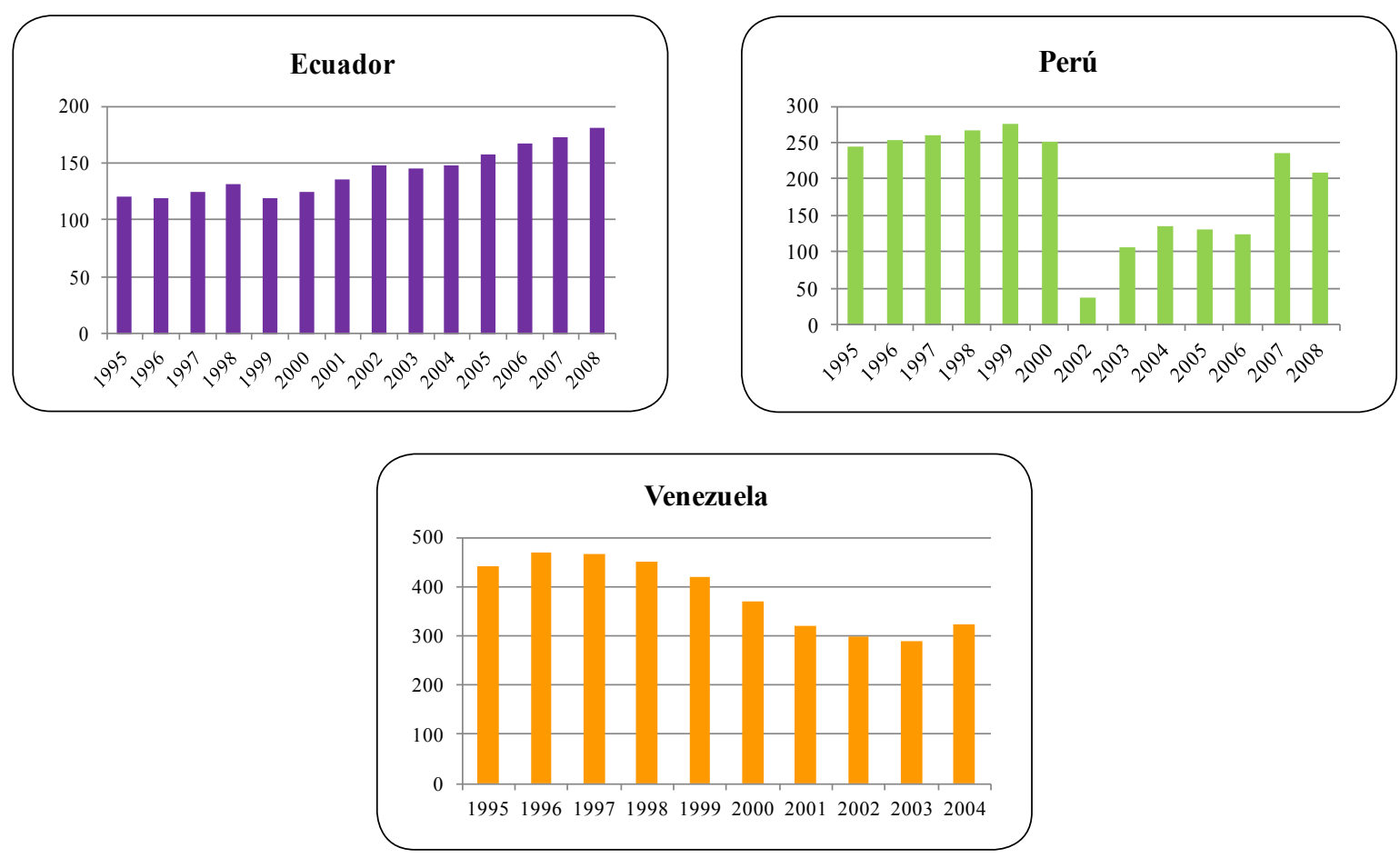

Fuente: elaboración propia con base en las Estadísticas estructurales de la industria manufacturera 1995-2008, CAN.

Con respecto a la FBKF en las cinco naciones suramericanas en estudio durante el 2005 y 2008 evolucionó favorablemente hasta alcanzar un crecimiento del $17,9 \%$ en el 2008 , ya para el año 2009, producto de la crisis mundial, la FBKF cae, pero se recupera en 2010, registrando $14,2 \%$ de crecimiento, según los indicadores económicos de la CAN (véase gráfica 6).

Gráfica 6

Formación bruta de capital fijo, 2005-2010p/ (tasa de variación anual de los valores constantes)

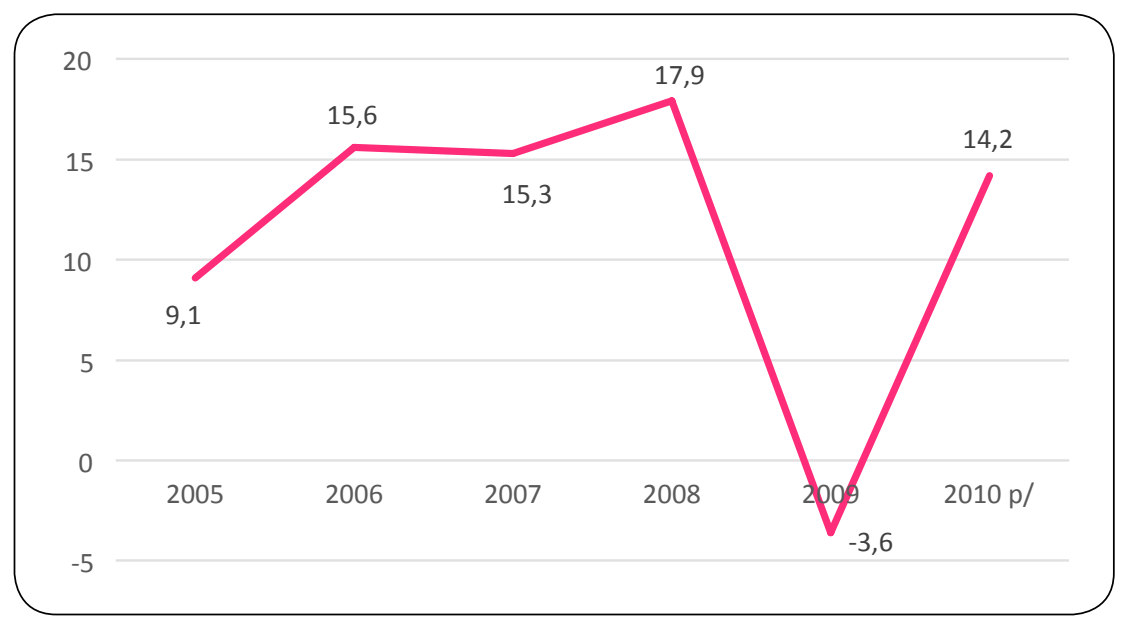

Nota: $\mathrm{p} /$ Cifra preliminar.

Fuente: elaboración propia con base en los Indicadores económicos 2011, CAN . 
Como se observa, el sector manufacturero ha sufrido fluctuaciones desde hace varios años, por motivo del lento crecimiento del PIB en la dinámica productiva, que conduce al mismo tiempo a la escasez de nuevos empleos en la economía; sin embargo, otras razones que afectan es la significativa participación de la industria en el producto nacional y el incremento de la FBKF hasta el 2008. Dado este panorama del sector, es de total interés estudiar la eficiencia de las aglomeraciones de estos países debido a que cada una de sus economías no logra ser lo suficientemente competitiva por sí sola.

\section{Aspectos de la modelación.}

En este artículo se proponen tres modelos para las estimaciones empíricas de la ET, que difieren entre sí según se incluya o no variables ambientales como argumento para identificar la realidad del sector. El primer modelo no comprende ninguna variable ambiental, por lo que se considera el más elemental. El segundo, incorpora la variable ambiental (VA1): FBKF, teniendo en cuenta la $\mathrm{ET}(\eta)$ y la variación del error en función de ineficiencia $(\gamma)$. El tercer modelo incluye la variable ambiental (VA2): FBKF en maquinaria y equipo, tanto en $\eta$ como en $\gamma$, no contiene la variable $V A 1$.

\section{Modelo 1.}

Las estimaciones iniciales de los parámetros de la función de producción translogarítmica de la ecuación (6) se presentan en la tabla $2^{11}$. Todos los coeficientes estimados son estadísticamente significativos con una confianza del $95 \%$, exceptuando el coeficiente del cambio tecnológico $(t)$, el cual está sujeto al tiempo. Los parámetros del modelo se denominan en este caso elasticidades de la producción con respecto a los insumos o elasticidades del producto-frontera orientado a los insumos (Battese \& Broca, 1997).

Considerando los parámetros estimados, se observa que $\beta_{0}, \beta_{2}, \beta_{4}, \beta_{6}$ y $\beta_{7}$ son mayores a cero mientras que $\beta_{1}, \beta_{3}, \beta_{5}, \beta_{8}$ y $\beta_{9}$ son menores a cero, lo cual implica un efecto positivo $\mathrm{y}$ negativo, respectivamente, de los cambios de las variables asociadas a estos coeficientes sobre el valor agregado en el sector manufacturero. Particularmente, el signo negativo de $\beta_{3}$ indica el deterioro de la tecnología en los catorce años de estudio. La elasticidad de la mano de obra estimada fue de 0,553 , lo cual indica que por un aumento del uno por ciento en la cantidad de trabajo, la producción manufacturera aumenta un $0,553 \%$, a diferencia de la elasticidad estimada con respecto al capital, ya que esta fue de $-0,87$, un impacto negativo sobre el nivel de producción.

De la misma forma, el efecto del cambio tecnológico que fue de $-0,165$. Lo anterior, puede obedecer al hecho de que este sector es intensivo en mano de obra, es decir, que las características del sector como tal, demandan más mano de obra para su producción que algún tipo de maquinaria o tecnología. Esto apunta a que la evolución del sector manufacturero depende sobre todo del uso de la mano de obra $(L)$; no obstante, las complementariedades tecnológicas parecen ser muy débiles todavía.

Aunque este primer modelo no tuvo en cuenta el término eta $(\eta)$ que indica si se mejoró la ET, el término gamma $(\gamma)$ estimado del 0,50, sí reveló que una parte de la variación del término de error $\left(\varepsilon_{i t}=V_{i t}-U_{i t}\right)$ se debe al componente de ineficiencia $\left(U_{i t}\right)$. 
Tabla 2

Estimación función producción translogarítmica, 1995-2008. Variable dependiente: logaritmo de la producción manufacturera

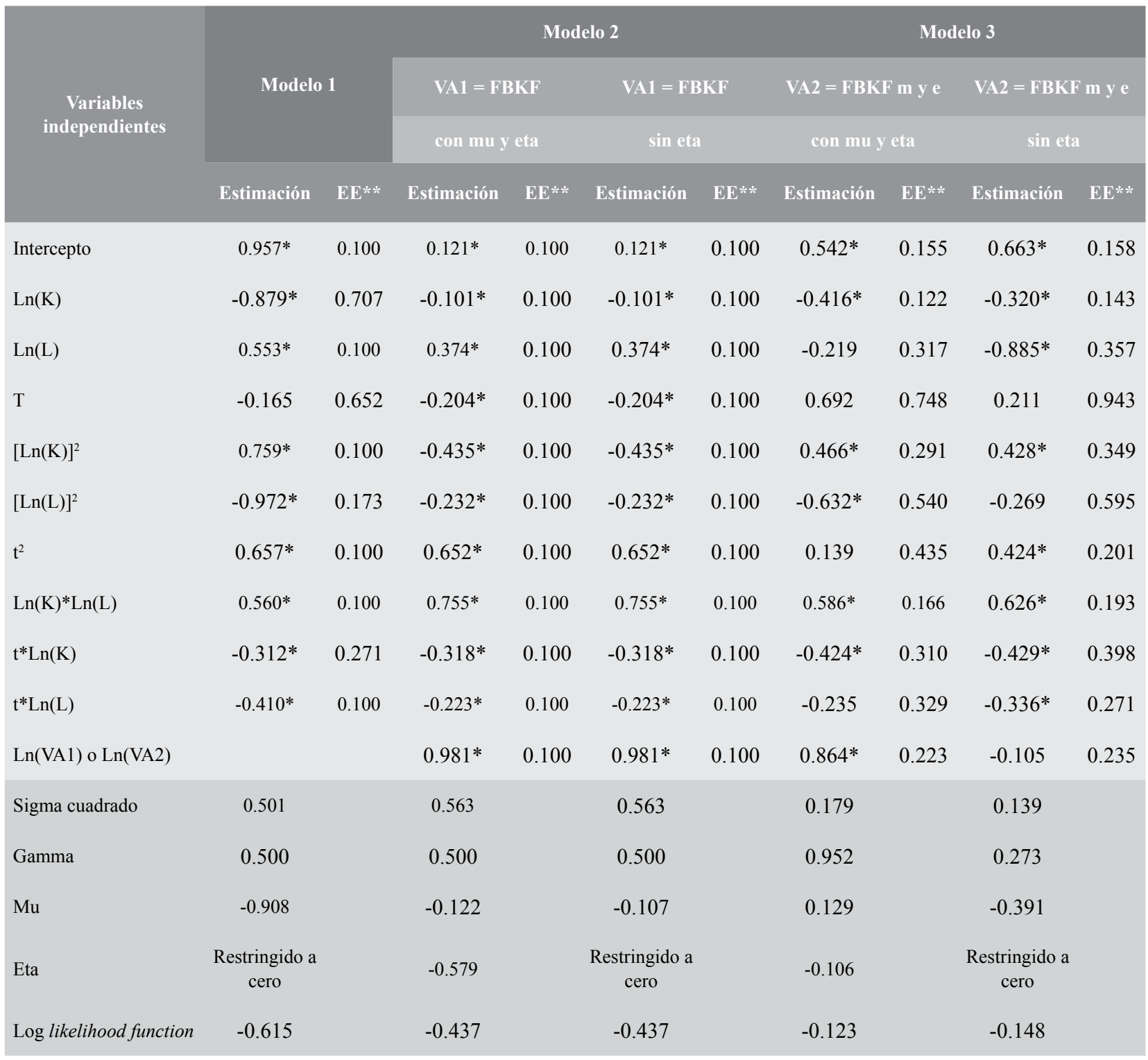

Nota: * Significativo al $5 \%$. EE: error estándar.

Fuente: elaboración propia.

\section{Modelo 2 y modelo 3.}

Por sí sola, la estimación de este modelo sería muy básica y poco confiable debido a la falta de variables ambientales y algunos términos de variación de la eficiencia, que reflejen el verdadero comportamiento del sector manufacturero. Es por esta razón que se estimaron dos modelos más, cuyos resultados se ilustran en la misma tabla 2, donde se tuvieron en cuenta variables ambientales como: la FBKF denominada $V A 1$ y la FBKF en maquinaria y equipo denominada $V A 2^{12}$. 
Adicionalmente, a cada variable ambiental se le modificó el parámetro de $\eta, \mathrm{y} \gamma$ que revela el mejoramiento de la ET y mide qué tanto del componente de ineficiencia técnica está compuesto el término del error.

De forma comparativa, entre los nuevos modelos, las estimaciones de los coeficientes de dichas variables ambientales $V A 1$ y VA2 se espera que tengan signos positivos, ya que se pueden considerar como una inversión que favorece a la producción y a su vez ayudan a disminuir la ineficiencia existente. Esto quiere decir, que entre mayor sea la inversión en el sector más productivo y eficiente podrá ser. Como se observa en la estimación del modelo 2, no todos los parámetros tienen los signos esperados, además no sufren muchas variaciones al cambiar los parámetros $\eta$ y $\gamma$ de la variable ambiental FBKF (VA1). En cuanto a la significancia de los coeficientes, los insumos resultaron ser todos significativos, incluso el cambio tecnológico que en el modelo 1 fue no significativo. Con respecto a la variable ambiental su signo positivo y significativo de 0,98 demuestra la relevancia de la inversión en la FBKF para el sector manufacturero.

La elasticidad conjunta insumo-producto de capital y mano de obra $\left(\operatorname{Ln}(K)^{*} \operatorname{Ln}(L)\right)$ y la de $V A 1$ son las que más contribuyen a agregarle valor al sector, es por eso, que un aumento en una unidad de estas, incrementa la producción en la industria en 0,755 y 0,981 , en su orden. A diferencia del modelo anterior, se incorpora el término $\eta$ correspondiente a $-0,57$ el cual su signo negativo indica que la ET no ha mejorado, aunque no hay evidencia de la existencia de cambios significativos en la eficiencia para los años estudiados. Con respecto a gamma estimado, revela que el componente de ineficiencia está contenido en la variación del término de error en un 0,50 .

Los parámetros estimados en el modelo 3 conservan los mismos signos del modelo 2 , sin embargo, para este caso, la modificación en los parámetros de gamma y eta conduce a que los valores de los coeficientes estimados cambien.
Referente a la significancia de los coeficientes, se debe realizar un análisis por separado, uno cuando se toma mu y eta, el otro sin eta.

El primero presenta significancia en las elasticidades de $K$ y $V A 2$, con excepción de la elasticidad de $L$, además su mayor aporte al valor agregado en la producción del sector está dado por la elasticidad de $V A 2$, la insumo-producto conjunta de capital y trabajo $(\operatorname{Ln}(K) * \operatorname{Ln}(L))$ y la insumo-producto respecto a capital $\left([\operatorname{Ln}(K)]^{2}\right)$ equivalentes a $0,864,0,586$ y 0,466 respectivamente. En el segundo, la elasticidad de $K$ y $L$ son significativas, pero la variable ambiental VA2 y el cambio tecnológico no lo son, ni con el $95 \%$ de confianza, su participación en la producción se refleja principalmente por la elasticidad insumoproducto conjunta del capital y mano de obra $(\operatorname{Ln}(K) * \operatorname{Ln}(L))$ con un incremento en 0,626 y en la elasticidad insumo-producto con respecto al capital $\left([\operatorname{Ln}(K)]^{2}\right)$ con un aumento de 0,428 .

En cuanto al término $\gamma$, para el primer caso, fue de 0,95 , un valor alto que permite evidenciar la gran participación que tiene el componente de ineficiencia en el término de error compuesto ${ }^{11}$, caso contrario ocurrió con el segundo, ya que su valor estimado es solo de 0,27 . Y para el otro término $(\eta)$, aunque dio un valor negativo de - 0,10 lo que significa la poca mejoría en la ET, esta es más positiva que la del modelo 2 que fue de $-0,57$. Por lo que revela que a los países del sector manufacturero todavía les faltan cosas por hacer en cuestión de eficiencia.

Por otro lado, en la tabla 3 se presenta la estimación de la ET promedio, calculada por año para los dos modelos. Como se puede observar, en el modelo 2 -el cual incluye variable ambiental FBKF y los términos $m u$ y eta- la ET promedio es de 87,5\%. En el modelo 3 -contiene la variable ambiental FBKF, esto es, solamente en maquinaria y equipo y los términos $m u$ y etala eficiencia es de $49 \%$. Lo que sugiere el poco impacto que esta última variable ambiental tiene en la tecnología de producción. 
Tabla 3

Estimación de la eficiencia técnica orientada al insumo con variables ambientales por año

\begin{tabular}{ccc} 
Año & $\begin{array}{c}\text { Modelo } 2 \\
\text { VA1 }=\text { FBKF* con } \\
\text { mu y eta }\end{array}$ & $\begin{array}{c}\text { Modelo } 3 \\
\text { VA2 }=\text { FBKF m y e } \\
\text { * con mu y eta }\end{array}$ \\
\hline 1995 & 0,8752 & 0,7311 \\
1996 & 0,8752 & 0,7076 \\
1997 & 0,8752 & 0,56024 \\
1998 & 0,8752 & 0,5249 \\
1999 & 0,8752 & 0,4883 \\
2000 & 0,8752 & 0,4506 \\
2001 & 0,8933 & 0,6384 \\
2002 & 0,8766 & 0,5427 \\
2003 & 0,8694 & 0,3341 \\
2004 & 0,8766 & 0,2955 \\
2005 & 0,8766 & 0,4527 \\
2006 & 0,8808 & 0,5065 \\
2007 & 0,8683 & 0,4724 \\
2008 & 0,8694 & 0,1554 \\
Promedio & $\mathbf{0 , 8 7 5 9}$ & $\mathbf{0 , 4 9 0 0}$ \\
\hline
\end{tabular}

Nota: *Eficiencia media por año (promedio de los cinco países). Fuente: elaboración propia.

Para terminar, de acuerdo con los resultados de la tabla 4, los niveles de ET del sector manufacturero en los países estudiados y en su conjunto, con el modelo 1 propuesto por Battese y Coelli (1992), son relativamente altos. Colombia es el país con mayor índice (91,6 \%), seguido por Ecuador $(90,4 \%)$ y Bolivia (89,9 \%). Ahora bien, al considerar las estimaciones a partir de los modelos 1 y 2 , el nivel promedio de ET para el conjunto sigue siendo alto $(88,2 \%$ y $99 \%$, respectivamente). No obstante, el último resultado (modelo 3) puede contener sesgos de estimación, puesto que para Venezuela hubo información faltante, aunque también cabe la posibilidad que la variable FBKF -maquinaria y equipo- tenga un menor peso en el total de FBKF, variable que tiene mayor impacto en la eficiencia.
Tabla 4

Estimación de la eficiencia técnica por modelo y país, 1995-2008

\begin{tabular}{lccc}
\multicolumn{1}{l}{ País } & Modelo 1 & Modelo 2 & Modelo 3 \\
Bolivia & 0,8991 & 0,8933 & 0,9992 \\
Colombia & 0,9165 & 0,8956 & 0,9992 \\
Ecuador & 0,9049 & 0,8910 & 0,9992 \\
Perú & 0,8480 & 0,8433 & 0,9992 \\
Venezuela & 0,8737 & 0,8868 & N. D. \\
Promedio & 0,8884 & 0,8820 & 0,9992 \\
\hline
\end{tabular}

Nota: N. D.: no disponible.

Fuente: elaboración propia.

\section{Conclusiones}

A pesar de las limitaciones de algunos datos como fue el caso de Venezuela, este trabajo expone una serie de resultados sobre la dinámica de la productividad del sector manufacturero de forma aglomerada e individual. De los cinco países en vía de desarrollo, tres poseen una máxima producción, realizando el mejor uso posible de los insumos de que disponen. A su vez, son eficientes técnicamente en el contexto individual, destacándose la industria colombiana.

La ET media por periodo no manifiesta mucha variación a través del tiempo en estudio -con VA1-. Esto puede deberse a que los niveles de productividad del sector tienden a ser más lentos en cuanto a su desarrollo tecnológico, más cuando se trata de naciones en vía de desarrollo, como las cinco que se examinaron aquí.

Para el tamaño del sector, se concluye que entre más grande sea, por lo general es más eficiente, ya que puede producir a mayor escala y aprovechar las economías de escala, como es el caso de Colombia, que su PIB manufacturero es el más alto al igual que su ET en comparación con los otros países. Por consiguiente, con el fin 
de mejorar la ET y lograr ser más competitivos, los otros países, sobre todo los más pequeños, deben acoger estrategias que les ayuden a incrementar su capacidad instalada.

En relación con los resultados de la estimación de los dos modelos que reflejan el mejor comportamientodel sectormanufacturero, es importante resaltar que la inversión en la tecnología o en la FBKF es fundamental en el desempeño de la industria. Por ende, al comparar los modelos se evidencia que tanto en lo individual como en el aglomerado son eficientes técnicamente, sin embargo por más que en el modelo 3 existe un sesgo en los datos o la posibilidad de que las demás variables que conforman el total de la FBKF tenga un impacto superior que solo la maquinaria y equipo, el componente de ineficiencia en el término de error de 0,95 y la mejoría en la ET de - 0,10 son más deseables, que el 0,27 de la participación de dicho componente en el error y el - 0,57 de la mejoría en la ET del modelo 2.

Este trabajo contribuye a evaluar la eficiencia del sector manufacturero de cinco países en vía de desarrollo en su conjunto. No obstante, es recomendable que en próximas investigaciones se analice el efecto de otros factores distintos a los incluidos en el estudio sobre la ET, en especial los que se relacionan con características de financiamientos, negociaciones de comercio, entre otros. Además, diversos estudios en otras economías han expuesto que los índices de eficiencia son parecidos independientemente de la metodología usada, se sugiere utilizar para futuras estimaciones análisis con técnicas no paramétricas y paramétricas y así evaluar la sensibilidad de los resultados encontrados en la industria ante los cambios de la metodología.

\section{Notas}

${ }^{1}$ Datos consolidados para el año 2008, tomados del Departamento Administrativo Nacional de Estadística y del Instituto Nacional de Estadística.
2 También es cierto que existen aspectos en los que estos países difieren. Su desarrollo económico ha sido heterogéneo por varios años. No obstante, desde el Acuerdo de Cartagena (1969) se ha manifestado la intensión de crecer unidos económicamente como comunidad. Esta diferencia es una de las causas que ha llevado a las migraciones y a caracterizarlos como naciones con mano de obra barata o mal remunera (Casas, 2001).

${ }^{3}$ La diversificación de la estructura productiva mejora la mezcla de productos y el vector de especialización internacional, es un determinante del cierre de la brecha de productividad respecto a la frontera tecnológica internacional y, por lo tanto, de la aceleración del crecimiento de la productividad agregada en economías abiertas. Tal diversificación produce en esencia no solo mejoras en la competitividad y el posicionamiento global, sino además permite mejores encadenamientos internos, lo que fortalece el impacto positivo del crecimiento económico sobre la productividad agregada de la economía (Vera, 2009).

${ }^{4}$ Este documento se concentrará principalmente en medidas de ET.

${ }^{5}$ Con los precios de los insumos se puede construir un indicador de la eficiencia de costos que lleva a una medida de la eficiencia de asignación (Coelli et al., 2005).

${ }^{6}$ Existen dos nociones de la función de producción: la teórica que manejan los ingenieros y la empírica que se creó a partir de los mejores resultados alcanzados en la práctica (Castro \& Salazar, 2011).

${ }^{7}$ La medición dirigida a la producción, refleja el óptimo nivel de productos que alcanza con unas cantidades de insumos. Entonces, la unidad de producción $A$ es ineficiente porque a lo largo de la frontera eficiente, que está determinada por la función de producción, conlleva usar mayor cantidad de insumos para 
obtener niveles de producción inferiores a estas, por más que use la misma cantidad de insumos que $B$. Siendo la unidad $B$ y $C$ eficientes (véase gráfica 2-a). Por otro lado, la medición orientada al producto se liga directamente con la frontera de posibilidades de producción (FPP), que expresa el conjunto máximo de producción dado un nivel de insumo. Por ende, se considera eficiente algún punto que se encuentra sobre la frontera (unidad $B$, gráfica 2-c); esto equivale a que la ET sea el ratio $\mathrm{OA} / \mathrm{OB}$. Caso contrario ocurre con la unidad $A$, donde un conjunto de insumos $X$ obtiene dos productos (output1, output 2) que al representarse en FPP se ubica por debajo de esta, lo que conduce a menores cantidades de producto empleando las mismas cantidades de insumos (Castro \& Salazar, 2011).

${ }^{8}$ La frontera de eficiencia se estima utilizando una función paramétrica o no paramétrica, usando métodos determinísticos o estocásticos. El primero, a cualquier desviación de la frontera lo clasifica como ineficiente y no permite la posibilidad de eventos aleatorios o de otros factores que afecten la producción, mientras que el segundo sí considera perturbaciones aleatorias. El SFA es una técnica de estimación no determinística.

${ }^{9}$ Battese y Coelli (1995) asumen que $U_{i t}$ se obtiene por el truncamiento de cero en la distribución normal con media $\delta Z_{\text {it }} \mathrm{y}$ varianza $\sigma_{u}{ }^{2}$. Donde $Z_{i t}$ denota al vector $(g x l)$ que contiene las variables específicas del país y se sospecha que estos factores contribuyen a la ineficiencia de la nación y $\delta$ es un vector $(1 x g)$ de coeficientes desconocidos. A su vez, $U_{i t}=$ $\delta Z_{i t}+W_{i t}$, donde $W_{i t}$ son las variables aleatorias truncadas con media cero y varianza $\sigma_{u}{ }^{2}$. Así, $U_{i t}$ está distribuida como $N+\left(\delta Z i t, \sigma_{u}{ }^{2}\right)$. En otras palabras, este truncamiento ocurre en el punto $\delta Z_{i t}$ de modo que $U_{i t}$ es no-negativa.

${ }^{10}$ Para mayor detalle sobre el procedimiento, véanse Sharma et al. (2007).
11 Los resultados de la estimación del modelo o ecuación (6) son los mismos cuando se tiene o no en cuenta el término $m u(\mu)$.

${ }^{12} \mathrm{La}$ estructura formal de la ecuación del modelo 2 y 3 está dada del siguiente modo:

Ecuación VA1: $\operatorname{Lnf}\left(\mathrm{x}_{\mathrm{i}}, \mathrm{t}, \beta\right)=\beta_{0}+\beta_{1} \operatorname{LnK}_{\mathrm{it}}$ $+\beta_{2} \operatorname{LnL}_{\text {it }}+\beta_{3} \mathrm{t}+0,5\left[\beta_{4}\left(\mathrm{LnK}_{\mathrm{it}}\right)^{2}+\beta_{5}\left(\mathrm{LnL}_{\mathrm{it}}\right)^{2}\right.$ $\left.+\beta_{6} t^{2}\right]+\beta_{7} \operatorname{LnK}_{\text {it }} \operatorname{LnL}_{\text {it }}+\beta_{8} \mathrm{LnK}_{\mathrm{it}}+\beta_{9} \mathrm{tLnL}_{\mathrm{it}}+$ $\beta_{10} \operatorname{LnVA} 1_{\text {it }}$.

Ecuación VA2: $\operatorname{Lnf}\left(\mathrm{x}_{\mathrm{it}}, \mathrm{t}, \beta\right)=\beta_{0}+\beta_{1} \operatorname{LnK}_{\mathrm{it}}$ $+\beta_{2} \mathrm{LnL}_{\mathrm{it}}+\beta_{3} \mathrm{t}+0,5\left[\beta_{4}\left(\mathrm{LnK}_{\mathrm{it}}\right)^{2}+\beta_{5}\left(\mathrm{LnL}_{\mathrm{it}}\right)^{2}+\right.$ $\left.\beta_{6} \mathrm{t}^{2}\right]+\beta_{7} \mathrm{LnK}_{\mathrm{it}} \mathrm{LnL}_{\mathrm{it}}+\beta_{8} \mathrm{tLnK}_{\mathrm{it}}+\beta_{9} \mathrm{tLnL}_{\mathrm{it}}+$ $\beta_{10} \operatorname{LnVA} 2_{\text {it }}$.

${ }^{13}$ Lo comprende el componente de error que explica el ruido estadístico y componente de error.

\section{Referencias}

Acevedo, M. (2004). Diferencias Regionales en la Eficiencia Técnica del Sector Confecciones en Colombia: Un análisis de Fronteras Estocásticas (Tesis de grado). Universidad de los Andes, Bogotá D.C.

Aigner, D., Lovell, C. A. K., \& Schmidt, P. (1977). Formulation and estimation of 25 stochastic frontier productions function models. Journal of economics, 6, 32-37.

Amézquita C. (2008). La industria manufacturera en Colombia 19952005. Revista Facultad de Ciencias Económicas: Investigación y reflexión, 16(2), 61-78.

Batra, G., \& Tan, H. (2003). SME Technical Efficiency and its Correlates: CrossNational Evidence and Policy Implications (Working Paper). Washington: World Bank Institute. Recuperado de http://info.worldbank.org/etools/docs/ 
library/74068/china/readings/oct30/ tan30engl.pdf

Battese, G., \& Broca, S. (1997). Functional Forms of Stochastic Frontier Production Functions and Models for Technical Inefficiency Effects: A Comparative Study for Wheat Farmers in Pakistan. Journal of Productivity Analysis, 8, 395-414.

Battese, G., \& Coelli, T. (1988). Prediction of firm level technical efficiencies with a generalized frontier production function and panel data. Journal of econometrics, 38, 387-399.

Battese, G., \& Coelli, T. (1992). Frontier production functions, technical efficiency and panel data with application to paddy farmers in Indian. Journal of productivity Analysis, 3(1), 153-169. doi: 10.1007/BF00158774

Battese, G., \& Coelli, T. (1995). A model for technical inefficiency effects in a stochastic frontier production function for panel data. Empirical Economics, 20, 325-332.

Brescia, V., Lema, D., \& Barrón, E. (2003). Dinámica de producción y eficiencia en empresas agrícolas: metodologías para el análisis de datos en panel (Instituto de Economía y Sociología - INTA, Documento de Trabajo 29). Recuperado de http://inta.gob.ar/sites/ default/files/script-tmp-dt29.pdf

Casas, A. M. (2001). La comunidad Andina: 30 años en busca del desarrollo. Revista de Fomento Social, 56(221), 65-91.

Castro, G., \& Salazar, R. J. (2011). Eficiencia financiera del sector industrial de agroquímicos, Colombia, 2005. Economía Gestión de desarrollo, 11, 91-119.
Caves, R. \& Barton, D. (1990). Efficiency in U.S. Manufacturing Industries. Journal of Economic Literature. 30(1), 185-187.

Coelli, T. (1996). A Guide to Frontier Version 4.1: A Computer Program for Frontier Production Function Estimation (Centre for Efficiency and Productivity -CEPA, Working Paper No. 96/07). Australia: University of New England.

Coelli, T., Rao, D. S. P., \& Battese, G. E. (1998). An introduction to efficiency and 26 productivity analysis. Boston: Kluwer Academic.

Coelli, T., Rao, D. S. P., \& Battese, G. E. (2005). An Introduction to Efficiency and Productivity Analysis (2da ed). Nueva York, Springer.

Debreu, G. (1951). The Coefficient of Resource Utilization. Econometrica, 19(3), 273-292.

Díaz, M., \& Sánchez, R. (2008). Firm size and productivity in Spain: A stochastic frontier analysis. Small Business Economics, 30, 315-323.

Farrell, M. J. (1957). The Measurement of Productive Efficiency. Journal of the Royal Statistical Society, 120(3), 253-90.

Green, A. \& Mayes, D. (1989). The measurement of technical inefficiency of UK manufacturing industry. Economic Journal, 10, 523-538.

Green, A., Harris, C. y Mayes D. (1991). Estimation of technical inefficiency in manufacturing industry. Applied Economics, 23(10), 1637-1647. doi: $10.1080 / 00036849100000128$ 
Grether, J. (1999). Determinants of Technological Diffusion in Mexican Manufacturing: A Plant-Level Analysis. World Development, 27, 1287-1298.

Harris, C. M. (1989). Technical in efficiency of Australian Manufacturing industry (Bureau of Industry Economics, Occasional Paper No. 4). Canberra: Bureau of Industry Economics

Kim, S. (2003). Identifying and Estimating Sources of Technical Inefficiency in Korean Manufacturing Industries. Contemporary Economic Policy, 21, 132-144.

Koopmans, T. C. (1951). Analysis of Production as an Efficient Combination of Activities. Activity Analysis of Production and Allocation. Nueva York: John Wiley \& Sons.

Kumbhakar, S. C., \& Lovell, C. A. K. (2000). Stochastic frontier analysis. Nueva York: Cambridge University Press.

Margono, H,. \& Sharma, S. (2006). Efficiency and Productivity Analyses of Indonesian Manufacturing Industries, Journal of Asian Economics, 17(6), 979-995.

Meeusen, W., \& Vanden Broeck, J. (1977). Efficiency estimation from Cobb-Douglas production functions with composed error. International economic review, 18, 284-299.

Mini, F., \& Rodríguez, E. (2000). Technical Efficiency Indicators in a Philippine Manufacturing Sector. International Review of Applied Economics, 14(4), 461-473.

Moreno, A. M. (2008). Las Leyes del Desarrollo Económico Endógeno de Kaldor: El caso Colombiano. Revista de Economía Institucional, 10(18), 129147. doi: 10.1080/02692170050150138
Mutis, H. (2006). Una aplicación del análisis de frontera estocástica: el caso de hospitales de nivel II en Colombia. Lecturas Matemáticas, Volumen Especial, 259-270.

Pareto, V. (1906). Manual of political economy (Trad. A. S. Schwier). Nueva York: Augustus M. Kelley.

Patiño Y. A., Gómez, G. A., Osorio, E. (2010). Evaluación del desempeño del sector de distribución de electricidad en Colombia: una aplicación del análisis de frontera estocástica. Ensayos sobre política económica, 28(62), 70-123.

Quintero, J. (2005). Competitividad en la industria manufacturera de Cartagena: Un análisis de eficiencia técnica en el periodo 2001-2004 (Serie de estudios sobre la competitividad en Cartagena). Cartagena: Observatorio del Caribe Colombiano. Recuperado de http:// www.ocaribe.org/publicacionesinfo. php?la=en\&id_publicacion $=73$

Sharma S.C., Sylwester K., \& Margono H. (2007). Decomposition of total factor productivity growth in U.S. states. The quarterly review of economics and finance, 47, 215-241.

Tansini, R., \& Triunfo, P. (1998). Eficiencia técnica y apertura comercial en cuatro ramas industriales (Universidad de la República. Documento de Trabajo No. 09/98). Recuperado de https://www.colibri.udelar.edu.uy/bitstream/123456789/2321/1/DT\%20

E\%201998-09.pdf

Vera, L. (2009). Declinación y potencialidades del sector industrial manufacturero en Venezuela (Tesis de maestría). Universidad central de Venezuela, Caracas. 